\title{
Nahrungsmittelallergien im Kindesalter
}

\author{
Food Allergy in Childhood
}

Autoren

Institut
R. Treudler, J. C. Simon

Klinik für Dermatologie, Venerologie und Allergologie, Universitätsklinikum Leipzig A.ö.R.

\section{Bibliografie}

DOI $10.1055 / \mathrm{s}-2007-995386$

Akt Dermatol 2007; 33:

466-471 @ Georg Thieme

Verlag KG Stuttgart · New York ISSN 0340-2541

Korrespondenzadresse

\section{Dr. Regina Treudler}

Klinik für Dermatologie,

Venerologie und Allergologie

Universitätsklinikum Leipzig

A.ö.R.

Philipp-Rosenthal-Str. 23-25

04103 Leipzig

Regina.Treudler@

medizin.uni-leipzig.de

\section{Zusammenfassung \\ $\nabla$}

Bei den Nahrungsmittelallergien im Kindesalter gibt es eine deutliche Diskrepanz zwischen der Eigenwahrnehmung durch die Betroffenen und deren Eltern und objektiv nachweisbaren Sensibilisierungen. Oft werden eigeninitiiert diätetische Manipulationen durch die Eltern vorgenommen. Evidenzbasiert wird zur Prävention atopischer Erkrankungen aktuell das Stillen über mindestens vier Monate empfohlen. Sollte dieses nicht möglich sein, so können konventionelle Kuhmilch-Formula und - nur bei hohem Risiko - die hypoallergenen Formula eingesetzt werden. Liegt eine manifeste atopische Dermatitis vor, so sollte der mögliche Einfluss von Nahrungsmittelallergien durch Anamnese, Haut- und In-vitroTeste sowie durch Eliminations- und Provokationsdiäten überprüft werden. Bei Vorliegen einer Kuhmilchallergie werden extensiv hydroly-

\section{Einfihrung}

$\nabla$

Nahrungsmittelallergien sind Unverträglichkeiten von Nahrungsmitteln auf immunologischer Basis und sind insbesondere für Symptome an der Haut, dem Gastrointestinaltrakt und dem Atemtrakt verantwortlich. Die genaue Prävalenz von Nahrungsmittelallergien bei Kindern ist unbekannt und wird mit ca. $6 \%$ geschätzt [1]. Viele Eltern bringen auch eine Reihe weiterer klinischer Symptome ihrer Kinder mit einer vermuteten Allergie auf Nahrungsmittel in Verbindung. Eine aktuelle Metaanalyse von verfügbaren Studien zeigte, dass etwa $12 \%$ aller Eltern bei ihren Kindern eine Nahrungsmittelallergie vermuten [2]. Noch höher ist die Anzahl bei Eltern von Kindern mit einer atopischen Dermatitis. Besonders häufig wurden hierbei Allergien auf Milch, Eier, Fisch, Schalentiere und Erdnüsse vermutet [2]. Diese selbst diagnostizierten Allergien lassen sierte hypoallergene Formula therapeutisch empfohlen. Die Notwendigkeit einer Diät sollte bei Kindern alle 12-24 Monate überprüft werden, da sich die Allergien bei den meisten Kindern verlieren. Pollen-assoziierte Nahrungsmittelallergien manifestieren sich bei Kindern in ähnlicher Art wie bei den Erwachsenen v.a. mit einem oralen Allergiesyndrom und insbesondere bei Birken- und Beifuß-Allergien. Bei der chronisch rezidivierenden Urtikaria sollte auch bei Kindern an eine Triggerung durch Pseudoallergene in Nahrungsmitteln gedacht werden, während bei der akuten Urtikaria und der Anaphylaxie insbesondere Erdnüsse, Baumnüsse und Kuhmilch als Auslöser eine Rolle spielen. Neben der Nahrungsmittelkarenz, deren Realisierung durch die Deklarationspflicht von bisher 14 Nahrungsmitteln unterstützt wird, ist bei schweren Fällen von Nahrungsmittel-Allergien die Verordnung von Notfall-Medikamenten erforderlich.

sich dagegen bei der allergologischen Diagnostik nur bei einem geringen Teil dieser Patienten mit einer immunologischen Sensibilisierung in $\mathrm{Zu}-$ sammenhang bringen. Noch weniger Patienten zeigen reproduzierbare Symptome nach Durchführung einer oralen Provokationstestung [2,3]. Insgesamt besteht der Eindruck, dass die Bedeutung von Nahrungsmittelallergien bei der Auslösung von Beschwerden von den Betroffenen bzw. deren Eltern überschätzt werden. Dieses führt nicht selten dazu, dass teils sehr einschränkende selbst initiierte Diäten durchgeführt werden, die bei Kindern zu Fehl- oder Mangelernährungen führen können. $75 \%$ aller Eltern von Kindern mit atopischer Dermatitis haben bereits mindestens einmal eine diätetische Maßnahme bei ihren Kindern ausprobiert, wobei 39\% von einem Erfolg berichtet [4]. Speziellen Diäten liegt nicht selten der Gedanke zu Grunde, dass ohne Allergen keine Sensibilisierung und damit keine Aller- 
gie auftritt. Dabei wird nicht bedacht, dass bei Nahrungsmitteln meist keine lebenslange Meidung möglich ist und dass ohne einen Kontakt zu potentiellen Allergenen auch keine Toleranzinduktion erfolgt .

Für den pädiatrisch-allergologisch tätigen Arzt ist es essenziell sich mit dem Thema von Nahrungsmittelallergien bei Kindern auseinanderzusetzen, um die Betroffenen und deren Eltern nach dem heutigen Erkenntnisstand zu beraten. Ziele einer möglichen diätetischen Intervention bei Kindern betreffen einerseits die Prävention der Manifestation atopischer Erkrankungen, andererseits die Therapie bekannter Nahrungsmittelallergien. Diese Übersichtsarbeit soll aktuelle Ansichten zum Einfluss von Nahrungsmitteln auf die Entwicklung einer atopischen Dermatitis sowie den diagnostischen und therapeutischen Möglichkeiten bei Nahrungsmittelallergien vom Sofort- und Spättyp wiedergeben.

\section{Pathogenese von Nahrungsmittel-Allergien}

Die Sensibilisierung auf Nahrungsmittelallergene erfolgt im frühen Kindesalter in der Regel über den Verdauungstrakt. Hier spielen die auch dem Typ 1 zugeordneten, hitze- und säurestabilen Allergene wie z. B. Kuhmilch, Hühnerei und Erdnuss eine Rolle. Klinisch kann es zur Verschlechterung einer atopischen Dermatitis kommen, wobei sowohl IgE-vermittelte als auch zelluläre Reaktionen vorkommen können. Zudem können - meist IgEvermittelte - Soforttypreaktionen im Sinne einer Urtikaria bis hin zur Anaphylaxie auftreten [5,6].

Ältere Kinder, Jugendliche und Erwachsene reagieren eher auf die dem Typ 2 zugeordneten - meist hitzelabilen - Allergene, bei denen die Sensibilisierung über die Atemwege erfolgt. Hierbei handelt es sich in erster Linie um IgE-vermittelte Reaktionen auf pollenassoziierte Nahrungsmittel. Bekannt ist insbesondere das Birken-Obst-Syndrom [5], bei dem meist ein orales Allergiesyndrom auftritt, das auch in seltenen Fällen eine Systembeteiligung bis hin zur Anaphylaxie zeigen kann $[5,6]$.

Von den Nahrungsmittelallergien abgegrenzt werden müssen diverse nicht-immunologisch bedingte Reaktionen auf Nahrungsmittel. So kann der Verzehr einiger Nahrungsmittelzusatzstoffe (v.a. Farb- und Konservierungsstoffe) zu einer nicht-immunologisch vermittelten Mastzellliberation führen, bei der die Symptome klinisch nicht von echten Allergien zu unterscheiden sind („Pseudoallergie“) [6]. Klassische Allergieteste versagen hier bei der Diagnostik. Zu den Allergien weiterhin abgegrenzt werden müssen Intoleranz-Reaktionen auf bestimmte Nahrungsmittel, die sich fast ausschließlich am Magen-Darm-Trakt abspielen und sich auf Enzymdefekte zurückführen lassen (z. B. Laktose-, Fruktose-, Histamin-Intoleranz). Auch muss bei persistierenden gastrointestinalen Beschwerden an das mögliche Vorliegen einer Mastozytose, die primär durch die Bestimmung der Tryptase im Serum untersucht werden kann, oder auch an chronisch entzündliche Darmerkrankungen gedacht werden. Nach Ausschluss organischer Ursachen für die Beschwerden sollte nicht zuletzt an eine psychosomatische Ursache gedacht werden.
Tab. 1 In Deutschland verfügbare hypoallergene Säuglingsnahrung (modifiziert nach [10])

\begin{tabular}{|c|c|c|c|}
\hline Produkt & $\begin{array}{l}\text { Protein- } \\
\text { quelle }\end{array}$ & $\begin{array}{l}\text { Hydrolyse- } \\
\text { grad }\end{array}$ & Indikation \\
\hline Pregestimil & Kasein & Extensiv & Therapie \\
\hline Alfaré & Molke & Extensiv & Therapie \\
\hline Milupa & $\begin{array}{l}\text { Soja/ } \\
\text { Kollagen }\end{array}$ & Extensiv & Therapie \\
\hline Nutramigen & Kasein & Extensiv & $\begin{array}{l}\text { Therapie/ } \\
\text { Prophylaxe }\end{array}$ \\
\hline Hipp pre HA, HA1, HA2 & Molke & Extensiv & Prophylaxe \\
\hline $\begin{array}{l}\text { Pre Aptamil HA } \\
\text { Aptamil HA1, HA2 }\end{array}$ & Molke & Extensiv & Prophylaxe \\
\hline Milumil HA1, HA2 & Molke & Partiell & Prophylaxe \\
\hline $\begin{array}{l}\text { Beba Start HA, PRE, } \\
\text { HA1, HA2, HA3 }\end{array}$ & Molke & Partiell & Prophylaxe \\
\hline Alete HA, HA 1, HA2 & Molke & Partiell & Prophylaxe \\
\hline Humana HA 1, HA2 & Molke & Partiell & Prophylaxe \\
\hline Milasan & Molke & Partiell & Prophylaxe \\
\hline Lactana HA1, HA2 & Molke & Partiell & Prophylaxe \\
\hline
\end{tabular}

\section{Diät zur Prävention atopischer Erkrankungen $\nabla$}

Bezüglich der Prävention atopischer Erkrankungen haben sich in den letzten Jahren eine Reihe von Hinweisen gezeigt, dass eine Gabe von Probiotika an Schwangere und Neugeborene die Frequenz des Auftretens eines atopischen Ekzems reduzieren kann [7]. Diese Daten ließen sich allerdings nicht in allen Studien reproduzieren. Eine reguläre Empfehlung zur Probiotikagabe lässt sich daher bis heute nicht ableiten. Ebenso gibt es keine sicheren Hinweise, dass eine sonstige mütterliche Diät während der Schwangerschaft sinnvoll zur Prävention atopischer Erkankungen eingesetzt werden kann [7]. Zur Primär- und Sekundärprävention atopischer Erkrankungen geben aktuelle Leitlinien folgende Empfehlungen $[8,9]$ :

1. Stillen über 4-6 Monate bei allen Kindern

2. Falls Stillen nicht möglich, Einsatz konventioneller Kuhmilch-Formula

3. Nur bei hohem Risiko (familiäre Vorbelastung) zur Sekundärprävention Einsatz möglichst extensiv hydrolysierter hypoallergener Formula-Diäten (sehr kostenintensiv).

Bei diesen hypoallergenen Formula Diäten handelt es sich um nahrungsmitteltechnisch behandelte Milch, bei der die Eiweiße (Kasein oder Molke) in unterschiedlichem Ausmaß durch enzymatische Spaltung, Ultraerhitzung und/oder Ultrafiltration aufgespalten sind, wodurch die Allergenität reduziert wird [10]. Nach dem Grad der Bearbeitung wird in partiell und extensiv hydrolysierte Formula unterschieden. Nach der Definition der American Academy for Paediatrics darf eine Formula-Diät als hypoallergen bezeichnet werden, wenn (1) die Antigene des Proteins reduziert sind, (2) sie als therapeutische Nahrung erfolgreich bei Patienten mit dokumentierter Proteinallergie eingesetzt wurde und (3) immunologische Reaktionen verhindert werden. In Deutschland sind diverse Produkte auf dem Markt ( Tab. 1) [10]. Es sei erwähnt, dass es zum Einsatz der hydrolysierten hypoallergenen Formuladiäten in der Prävention durchaus kontroverse Ansichten gibt, wobei darauf hingewiesen wird, dass gute, Evidenz basierte Daten fehlen, um tatsächlich einen langfristigen Erfolg auf die Prävention atopischer Erkrankungen zu zeigen [11]. 
Im weiteren Verlauf sollte bei familiärer Vorbelastung für atopische Erkankungen keine Beikost vor dem 4.-6. Lebensmonat erfolgen und danach die Beikost auch nur langsam ergänzt werden, maximal unter Hinzufügung eines Lebensmittels pro Woche. In der Regel wird hier mit Karottenmus begonnen und mit Kartoffeln sowie magerem Fleisch (Geflügel, Lamm, Kalb, Rind) fortgesetzt. Später kommen weitere verträgliche Gemüse hinzu (z. B. Blumenkohl, Brokkoli, Kohlrabi, Zucchini, Fenchel). Ein Zusatz von hochwertigem Speiseöl (Raps-, Sonnenblumen-, Maiskeimöl) sichert die ausreichende Versorgung mit wichtigen Fettsäuren. Eine langfristige präventive Meidung von Kuhmilch wird aufgrund des hohen ernährungsphysiologischen Wertes der Milch für nicht gerechtfertigt erachtet. Die Einführung sollte im Einzelfall maximal auf den neunten Lebensmonat hinausgezögert werden. Aufgrund des hohen Allergenpotenzials sollten Fisch, Hühnereier und Nüsse in den ersten ein bis zwei Jahren gemieden werden [12]. Weitere allgemeine Diätempfehlungen zur Prävention atopischer Erkrankungen lassen sich aktuell nicht sicher geben.

\section{Nahrungsmittelallergie bei Kindern mit atopischer Dermatitis \\ $\nabla$}

In einem aktuellen Positionspapier der European Academy for Allergy and Clinical Immunology [13] wird die Häufigkeit einer Nahrungsmittelallergie bei Kindern mit atopischem Ekzem mit zwischen 37 und $63 \%$ angegeben, wobei sich klinisch nicht nur ekzematöse Reaktionen, sondern auch nicht-ekzematöse Soforttyp-Allergien oder kombinierte Reaktionen zeigen können. Die Diagnostik einer solchen Nahrungsmittelallergie ist nicht selten sehr aufwändig [12,13]: Zunächst sollte die Anamnese einen möglichen Zusammenhang der Nahrungsmitteleinnahme mit einsetzenden Ekzemschüben beurteilen. Hierfür eignet sich auch das Führen eines Symptom- und Nahrungsmitteltagebuches. Zudem kann das spezifische IgE für einzelne Nahrungsmittel und das Gesamt-IgE zur Abschätzung des Atopiestatus bestimmt werden. Der Pricktest ist ein weiteres Standard-Diagnoseverfahren, wobei möglichst native Nahrungsmittel eingesetzt werden sollten. Der Atopie-Patch-Test bei der Abklärung von Nahrungsmittelallergien ist in der Regel zeitaufwändig, bringt nur geringe Zusatzinformationen und kann die orale Provokationstestung nicht ersetzen, die letztendlich über die klinische Relevanz nachgewiesener Sensibilisierungen entscheidet [14]. Insgesamt wird zur Diagnostik von Nahrungsmittelallergien bei der atopischen Dermatitis ein schrittweises Vorgehen zur Provokationstestung empfohlen ( $\bullet$ Tab. 2). Eine parallel zur Provokation durchzuführende oligoallergene Basisdiät sollte sich zusammensetzen aus Reis, Lamm, Pute, Blumenkohl, Brokkoli, Gurke, Mineralwasser, schwarzem Tee, milchfreier Margarine, raffiniertem Pflanzenöl, Salz, Zucker [12]. Repetitive orale Provokationsteste sollten Plazebo-kontrolliert erfolgen und verdächtige Nahrungsmittel sollten in einer festgelegten Reihenfolge zugeführt werden. Eingeschlossen werden häufig Kuhmilch, Hühnerei, Weizen, weitere Gemüsesorten, Obstsorten, Getreidesorten, Fleischsorten, Nüsse, Sellerie, Gewürze. Durch dieses sehr aufwändige Verfahren lässt sich ggf. die Relevanz von Nahrungsmitteln bei der Triggerung der atopischen Dermatitis zeigen, sodass eine Karenzdiät empfohlen werden kann [12,13]. Das Maximum der Nahrungsmittelallergien liegt im 2. bis 3. Lebensjahr. Wichtig ist, dass im Folgenden die meisten Kinder - es werden Daten bis zu $80 \%$ bis zum Schulalter genannt - eine Nahrungs-
Tab. 2 Diagnostische Schritte bei der Abklärung von Nahrungsmittelallergien vom Sofort- und vom Spättyp mittels oraler Provokationstestung bei Kindern mit persisterendem moderatem bis schwerem atopischem Ekzem (modifiziert nach [13])

Anamnese einer möglichen Nahrungsmittelallergie
Bestimmung des spezifischen IgE, Pricktest (evtl. Atopie-Patchtest)
Diagnostische Eliminationsdiät über einen Zeitraum von 4 - 6 Wochen
in einer stabilen Phase der Erkrankung Evaluation des Ekzem-Scores vor
der OPT
Titrierte OPT
Evaluation von nicht-ekzematösen Symptomen während der Titration
und der folgenden zwei Stunden
Evaluation des Ekzem-Scores 16 - 24 Stunden nach OPT
Bei negativem Befund: Wiederholung der OPT mit der durchschnitt-
lichen täglichen Menge des Nahrungsmittels über einen Zeitraum von
mehreren Tagen, dabei täglich Evaluation des Ekzem-Scores
Ggf. nach einem freien Tag Fortsetzung mit einem anderen Nahrungs-
mittel

mittelsensibilisierung wieder verlieren. Bezüglich der Kuhmilchallergie konnte allerdings kürzlich in einer Untersuchung an 807 Kindern eine insgesamt schlechtere Prognose gezeigt werden. So verloren $19 \%$ der Kinder die Sensibilisierung bis zum vierten, $42 \%$ bis zum achten, $4 \%$ bis zum zwölften und erst $79 \%$ bis zum 16 . Lebensjahr [15].

Hieraus leitet sich die Empfehlung ab, dass Karenzdiäten im Kindesalter jeweils nur für 12 bis 24 Monate gültig sein sollten. Im Anschluss sollte jeweils erneut eine mögliche Verträglichkeit überprüft werden. Um eine langfristig notwendige Karenzdiät von bestimmten Nahrungsmitteln zu umgehen, wird aktuell in Studien eine selektive orale Toleranzinduktion (SOTI) versucht [16], bei der durch regelmäßiges orales Zuführen zunächst kleiner und dann größerer Mengen des Allergens - meist Kuhmilch - zumindest bei einem Teil der Kinder eine Gewöhnung an das Allergen herbeigeführt werden kann. Zur endgültigen Beurteilung dieses sehr aufwändigen Verfahrens fehlen noch Daten von größeren Kollektiven.

\section{Orales Allergie-Syndrom $\nabla$}

Das orale Allergiesyndrom, das eher bei älteren Kindern und Jugendlichen auftritt, geht vor allem mit Missempfindungen im Mundbereich einher [3]. Selten kann es auch zu Schwellungen im Mund-/Rachenbereich sowie zu generalisierten Symptomen kommen. Es handelt sich in der Regel um eine immunologische Kreuzreaktion bei vorbestehender Pollensensibilisierung (insbesondere bei Birken- und Beifuß-Sensibilisierten, 0 Tab.3), wobei Sequenz-, Peptid- und Proteinhomologien zwischen den Pollenallergenen und insbesondere Kern- und Steinobstfrüchten verantwortlich sind [5]. So ähnelt das Protein des Hauptallergens der Birke, Bet v 1, dem Haselnuss-Allergen Cor a 1 zu 79\%, dem Apfel-Allergen Mal d 1 zu 71\% und dem Soja-Allergen Gly m 4 zu $63 \%$ [5,17]. Bedeutsam ist, dass bei solchen kreuzallergisch bedingten Nahrungsmittelallergien bereits der Erstkontakt mit dem Nahrungsmittel zu allergischen Symptomen führen kann, ohne dass sich der Patient dieses Risikos bewusst ist. Dieses hat in den letzten Jahren insbesondere bei Birkenpollenallergikern nach Verzehr der sehr beliebt gewordenen Sojamilch-Produkte, bei denen zudem noch sehr rasch eine große Menge des Proteins zugeführt wird, zu schweren anaphylaktischen Reaktionen geführt [18]. 


\begin{tabular}{|c|c|c|c|c|}
\hline $\begin{array}{l}\text { Inhalationsallergene } \\
\text { und Häufigkeit einer } \\
\text { assoziierten NMA (\%) }\end{array}$ & Nahrungsmittel & $\begin{array}{l}\text { Sensibilisierung } \\
\text { auf das NM (\%) }\end{array}$ & $\begin{array}{l}\text { Manifeste Allergie } \\
\text { auf das NM (\%) }\end{array}$ & $\begin{array}{l}\text { Tab. } 3 \text { Pollenassoziierte } \\
\text { Nahrungsmittelallergien } \\
\text { (modifiziert nach [5], } \\
\text { NMA Nahrunasmittelalleraie: }\end{array}$ \\
\hline \multirow{9}{*}{$\begin{array}{l}\text { Baumpollen } \\
(30-93 \%)\end{array}$} & Apfel & $80-90$ & $50-65$ & \multirow{15}{*}{$\begin{array}{l}\text { NM, Nahrungsmittel; } \\
\text { K. A., keine Angabe) }\end{array}$} \\
\hline & Haselnuss & $70-80$ & $40-60$ & \\
\hline & Pfirsich, Nektarine & K. A. & $20-30$ & \\
\hline & Kirsche & K. A. & $20-25$ & \\
\hline & Kiwi & K. A. & 10 & \\
\hline & Karotte & K. A. & 9 & \\
\hline & Sellerie & 50 & $0-20$ & \\
\hline & Kartoffel & K. A. & 7 & \\
\hline & Soja & 70 & 10 & \\
\hline \multirow{6}{*}{$\begin{array}{l}\text { Beifußpollen } \\
(16-27 \%)\end{array}$} & Sellerie & 52 & 42 & \\
\hline & Mango & K. A. & 29 & \\
\hline & Gewürze & 45 & 14 & \\
\hline & Sonnenblumensamen & K. A. & 14 & \\
\hline & Weintraube & K. A. & 14 & \\
\hline & Litschi & K. A. & 7 & \\
\hline
\end{tabular}

In einer Studie von Roehr et al. [3] wurden 2345 zufällig ausgewählte Berliner Kinder mittels eines Fragebogens zu Nahrungsmittelallergien befragt. 739 Kinder bzw. deren Eltern antworteten, wobei 14,1\% der 1-17-Jährigen orale Missempfindungen, insbesondere nach dem Verzehr von Apfel und Haselnuss angaben, was in etwa der Häufigkeit des Vorkommens bei Erwachsenen entspricht.

\section{Nahrungsmittel-Unverträglichkeit bei der Urtikaria}

Bei der akuten Urtikaria spielen bei Kindern nicht selten IgE-vermittelte Mechanismen auf Nahrungsmittel eine Rolle, wobei es auch zum Auftreten weiterer Allgemeinsymptome bis hin zur Anaphylaxie kommen kann (siehe unten). Bei der chronisch rezidivierenden Urtikaria dagegen treten eher nicht-immunologisch vermittelte Reaktionen auf Nahrungsmittelzusatzstoffe in den Vordergrund. Hierbei werden sogenannte Pseudoallergene z.B. Farb- (Tartrazin, Azorubin u.a.), Konservierungsstoffe (Sorbinsäure, Natriumbenzoat, Hydroxybenzoesäure u.a.), Geschmacksverstärker (Natriumglutamat), Antioxidanzien (Tocopherol, Propylgallate u.a.) - für die Freisetzung von Mediatoren aus Mastzellen verantwortlich gemacht [19]. In einer Untersuchung von Ehlers et al. [20] konnten bei 12 von 16 (75\%) Kindern mit chronisch rezidivierender Urtikaria durch eine Eliminationsdiät und anschließende doppelblinde Plazebo-kontrollierte Provokation Pseudoallergene als Auslöser identifiziert werden. Dieses liegt sogar noch etwas über den Werten, die bei Erwachsenen gefunden werden, sollte aber aufgrund der kleinen Fallgruppe nicht überinterpretiert werden. Wichtig ist, dass sich diese Pseudoallergien nicht durch Routinelaboruntersuchungen oder Hautteste diagnostizieren lassen. Stattdessen sollte bei entsprechendem Verdacht auf jeden Fall eine Eliminationsdiät unter Verzicht auf Pseudoallergene - und eventuell im Anschluss eine Provokationstestung zur diagnostischen Sicherung - durchgeführt werden. Ein Erfolg tritt in der Regel erst nach 2 bis 3 Wochen Eliminationsdiät auf. Hier ist also genug Geduld erforderlich, bevor die Rolle von Pseudoallergenen auf die Urtikaria beurteilt werden sollte.

\section{Anaphylaxie \\ $\nabla$}

Die schwerste Form der allergischen Soforttypreaktion ist die Anaphylaxie, wobei bisher Uneinigkeit über feste Diagnosekriterien herrscht. Zur Schweregrad-Einteilung wird im deutschsprachigen Raum meist die Einteilung nach Ring und Messmer verwendet [21]. Das kürzlich unter Beteiligung deutschsprachiger Allergiezentren gegründete Anaphylaxie-Register (NORA-Network for Online Registration of Anaphylaxis) hat sich zum Ziel gesetzt, valide Daten zu Epidemiologie, Klinik und Auslösern anaphylaktischer Reaktionen zu sammeln, die erstaunlicherweise bis heute für Deutschland fehlen [22]. Aus anderen Ländern ist bekannt, dass bei Kindern Nahrungsmittel noch vor den Insektenstichen und den eher seltenen Reaktionen auf Medikamenten zu den häufigsten Auslösern gehören [23]. Eine in Deutschland durchgeführte Fragebogen-Untersuchung, bei der 103 Anaphylaxie-Fälle von Mehl et al. (Allergy 2005) retrospektiv ausgewertet wurden, zeigte, dass Nahrungsmittel bei $57 \%$ der Kinder als Auslöser identifiziert wurden [24]. Hierbei handelte es sich in etwa zu gleichen Teilen um Erdnuss, Baumnuss und Milch. Etwas weniger häufig genannt wurden Fisch und Hühnerei. In anderen Ländern, insbesondere in den USA, spielt die Erdnuss eine noch größere Rolle als Auslöser schwerer allergischer Reaktionen [23]. Anaphylaktische Reaktionen können einen biphasischen Verlauf zeigen, wobei es nach ursprünglicher Besserung zum Wiederauftreten der Symptome kommen kann. Insofern sollte im Anschluss an eine Akutversorgung eine 24-stündige Nachbeobachtung des Kindes durchgeführt werden [25]. Bei Kindern, die eine anaphylaktische Reaktion erlitten haben, sollten alle geeigneten Maßnahmen (Haut-/Blutteste/ggf. Expositionsteste) zur Identifikation der Auslöser unternommen werden.

\section{Therapie der Nahrungsmittelallergie}

Bei nachgewiesener Nahrungsmittelallergie ist es entscheidend, dass die betroffenen Kinder, die Eltern sowie auch ggf. verantwortliche Betreuer wie Großeltern, Erzieher und Lehrer gut informiert werden, um sicherzustellen, dass für das Kind ein erneuter Kontakt zu den Allergenen vermieden wird, bzw. um sicherzustellen, dass ggf. geeignete Notfall-Maßnahmen durchgeführt werden können. Notfallmedikamente sollten zur Verfügung gestellt werden und dabei folgende Präparategruppen um- 
Tab. 4 Beispiel für Notfall-Medikation bei Kindern bestehend aus einem oralen Antihistaminikum, einem oralen Glukokortikosteroid, einem inhalativen Bronchodilatator und einem Adrenalin-Präparat zur Selbstinjektion

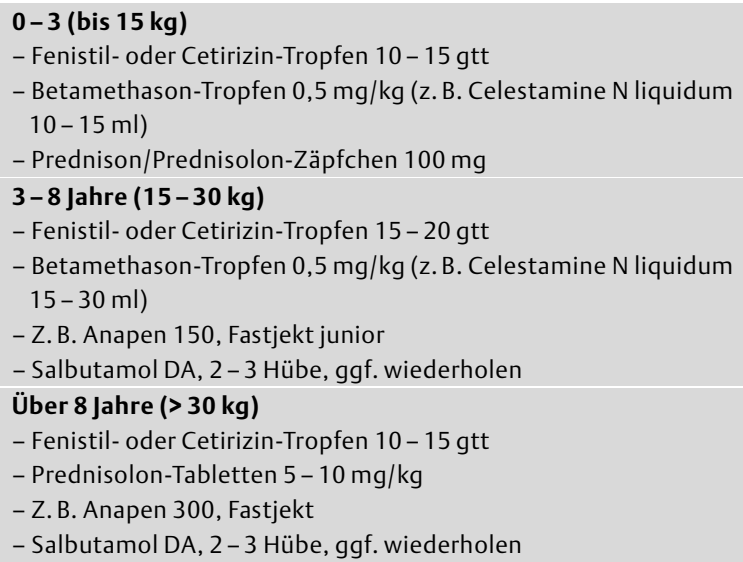

Tab. 5 Deklarationspflichtige Nahrungsmittel in Deutschland (betrifft nur abgepackte Ware) [28]

Glutenhaltiges Getreide: Weizen, Roggen, Gerste, Hafer, Dinkel, Kamut oder Hybridstämme davon sowie daraus hergestellte Erzeugnisse Eier und Eiererzeugnisse

\section{Erdnüsse und Erdnusserzeugnisse}

Nüsse: Mandel, Haselnuss, Walnuss, Cashewnuss, Pecannuss, Paranuss, Pistazie, Macadamianuss, Queenslandnuss sowie daraus hergestellte Erzeugnisse

Soja und Sojaerzeugnisse

Senf und Senferzeugnisse

Krebstiere und Krebstiererzeugnisse

Fisch und Fischerzeugnisse

Milch und Milcherzeugnisse (einschließlich Laktose)

Sellerie und Sellerieerzeugnisse

Schwefeldioxid und Sulfite in einer Konzentration von mehr als

$10 \mathrm{mg} / \mathrm{kg}$ oder $10 \mathrm{mg} / \mathrm{l}$ als So 2 angegeben

Sesamsamen und Sesamsamenerzeugnisse

Mollusken (Muscheln, Tintenfisch)*

Lupine*

${ }^{*}$ Neu seit 23. 12. 2007

fassen: (1) ein orales Antihistaminikum, (2) ein orales Glukokortikosteroid, (3) ggf. einen inhalativer Bronchodilatator (insbesondere bei gleichzeitig bestehendem Asthma) und - bei vorbeschriebenen starken Reaktionen - (4) ein Adrenalin-Präparat zur Selbstinjektion (siehe Tab. 4) [26,27]. Was die Allergenkarenz betrifft, so wird diese dadurch erleichtert, dass mittlerweile immerhin aktuell die 14 häufigsten Nahrungsmittelallergene deklarationspflichtig sind ( Tab.5). Allerdings betrifft diese Deklarationspflicht bisher nur abgepackte Ware und die Deklaration kann sich ggf. auch nur auf Umverpackungen befinden. Zudem haben Patienten mit selteneren Nahrungsmittel-Allergien noch keine Möglichkeit, zuverlässig eine Allergenmeidung herbeizuführen. Hier besteht noch weiterer Handlungsbedarf.
Abstract

\section{Food Allergy in Childhood}

$\nabla$

There is a discrepancy between self-reported and pysician-diagnosed food hypersensitivity in children, and parents tend to selfinitiated dietary interventions.

Evidence based guidelines for prevention of atopic diseases recommend breast feeding for at least four months. Conventional cow mild formula should be used if breast feeding is not possible. Only in high risk children, hypoallergenic formula are recommended. In children with atopic dermatitis, the possible influence of food allergies has to be investigated by history, skin and in vitro tests as well as by elimination and/or provocation diet. Children allergic to cow milk should be fed with extensively hydrolysed hypoallergenic formula. As most children lose their food allergy, the necessity of interventional diets has to be reevaluated every 12-24 month. Pollen-associated food allergies in children do not differ from those in adults and lead to oral allergy syndrome, especially in those with sensitizations to birch or mugwort. Pseudoallergens may trigger symptoms in children with chronic idiopathic urticaria, while in acute urticaria and in anaphylaxis, peanuts, tree nuts and cow milk are the main eliciting factors.

Therapeutically, food avoidance is essential, which is facilitated by the duty of declaration regarding the 14 most frequent food allergens. In severe cases of food allergy, emergency kits have to be provided.

\section{Literatur}

1 Sicherer SH, Sampson HA. Food allergy. J Allergy Clin Immunol 2006; 117: $470-475$

2 Rona RJ, Keil T, Summers C, Gislason D, Zuidmeer L, Sodergren E, Sigurdardottir ST, Lindner T, Goldhahn K, Dahlstrom J, McBride D, Madsen C. The prevalence of food allergy: a meta-analysis. J Allergy Clin Immunol 2007; 120: 638-646

3 Roehr CC, Edenharter G, Reimann S, Ehlers I, Worm M, Zuberbier T. , Niggemann B. Food allergy and non-allergic food hypersensitivity in children and adolescents. Clin Exp Allergy 2004; 34: 1534 - 1541

4 Johnston GA, Bilbao RM, Graham-Brown RAC. The use of dietary manipulation by parents of children with atopic dermatitis. Brit J Dermatol 2004; 150: 1186- 1189

5 Kleine-Tebbe J, Herold DA. Kreuzreaktive Allergenkluster bei pollenassoziierter Nahrungsmittelallergie. Hautarzt 2003; 54: 130-137

6 Averbeck M, Gebhardt C, Emmrich F, Treudler R, Simon JC. Immunologische Grundlagen der Allergien. J Dtsch Dermatol Ges 2007; 5: 10151028

7 Kramer MS, Kakuma R. Maternal dietary antigen avoidance during pregnancy or lactation, or both, for preventing or treating atopic disease in the child. Cochrane Database Syst Rev 2006; 19; 3: CD000133

8 Schäfer T, Borowski C, Diepgen TL, Hellermann M, Piechotowsik M, Reese I, Roos T, Schmidt S, Sitter H, Werfel T, Gieler U. Allergieprävention S3-Leitlinie. Allergo J 2004; 13: $252-260$

9 Werfel T, Fuchs T, Reese I, Erdmann S, Henzgen M, Kleine-Tebbe J, Lepp U, Niggemann B, Saloga J, Vieluf I, Vieths S, Zuberbier T. Vorgehen bei vermuteter Nahrungsmittelallergie bei atopischer Dermatitis. Allergo J 2002; 11: 386-393

10 Berg A von, Berdel D. Hypoallergene Säuglingsnahrung zur Allergieprävention. Monatsschr Kinderheilkd 2006; 154: 417-423

11 Brand PLP, Vlieg-Boerstra BJ, Dubois AEJ. Dietary prevention of allergic disease in children: Are current recommendations really based on good evidence? Ped Allergy Immunol 2007; 18: 475 - 479

12 Werfel T, Fuchs T, Reese I, Erdmann S, Henzgen M, Kleine-Tebbe J, Lepp U, Niggemann B, Saloga J, Vieluf I, Vieths S, Zuberbier T. Therapiemöglichkeiten bei der IgE-vermittelten Nahrungsmittel-Allergie. Allergo J 2002; $11: 156-162$ 
13 Werfel T, Ballmer-Weber B, Eigenmann PA, Niggemann B, Rancé F, Turjanmaa $K$, Worm $M$. Eczematous reactions to food in atopic a eczema: position paper of the EAACI and GA²LEN. Allergy 2007; 62: 723-728

14 Mehl A, Rolick-Werninghaus C, Staden U, Verstge A, Wahn U, Beyer K, Niggemann $B$. The atopy patch test in the diagnostic work up of suspected food-related symptoms in children. J Allergy Clin Immunol 2006; 118: $923-923$

15 Skripak JM, Matsui EC, Mudd K, Wood RA. The natural history of IgEmediated cow's milk allergy. J Allergy Clin Immunol 2007; 120: 1172 1177

16 Staden U, Rolinck-Werninghaus C, Brewe F, Wahn U, Niggemann B, Beyer K. Specific oral tolerance induction in food allergy in children: efficacy and clinical patterns of reaction. Allergy 2007; 62: 1261 1269

17 Jahn-Schmid et al. Bet v 114-156 is the dominant T-cell epitope of the major birch pollen allergen and important for cross-reactivity with Bet v 1-related food allergens. JACI 2005; 11: $213-219$

18 Süß A, Rytter M, Sticherling $M$, Simon JC. Anaphylaktische Reaktion auf ein Sojagetränk bei drei Patienten mit Birkenpollenallergie. JDDG; 2005; 3: 895 - 897

19 Zuberbier T, Edenharter G, Worm M, Ehlers I, Reimann S, Hantke T, Roehr CC, Bergmann KE, Niggemann B. Prevalence of adverse reactions to food in Germany - a population based study. Allergy 2004; 59: 338 345
20 Ehlers I, Niggemann B, Binder C, Zuberbier T. Role of nonallergic hypersensitivity reactions in children with chronic urticaria. Allergy 1998 Nov; 53: $1074-1077$

21 Treudler R, Kozovska Y, Simon JC. Severe immediate type reactions in 105 German adults: When to diagnose anaphylaxis. J Investigat Allerg Clin Immunol, im Druck

22 Hompes S, Kirschbaum J, Przybilla B, Rietschel B, Scherer K, Henzgen K, Treudler R, Hillen U, Mahler V, Worm M. Anaphylaxis register in Central Europe: first results. Allergy 2007; 62 (Suppl 83): 62

23 Roberts G. Anaphylaxis to foods. Ped Allergy Immunol 2007; 18: 543 548

24 Mehl A, Wahn U, Niggemann B. Anaphylactic reactions in children - a questionnaire based survey. Allergy 2005; 60 (11): 1440-1445

25 Lee JM, Greenes DS. Biphasic anaphylactic reactions in pediatrics. Pediatrics 2000; 106: $762-766$

26 Muraro A, Roberts G, Clark A, Eigenmann PA, Halken S, Lack G, MoneretVautrin A, Niggemann B, Rancé $F$. The management of anaphylaxis in childhood: position paper of the European academy of allergology and clinical immunology. Allergy 2007; 62: 857-871

27 Ring J, Brockow K, Duda D, Eschenhagen T, Fuchs T, Huttegger I, Kapp A, Klimek L, Müller U, Niggemann B, Pfaar O, Przybilla B, Rebien W, Ruëff F, Schnadt S, Tryba M, Worm M, Sitter H, Schultze-Werni G. Akuttherapie anaphylaktischer Reaktionen - S2-Leitlinie. Allergo J 2007; 6: 420434

28 Amtsblatt der Europäischen Union: „Zutaten im Sinne des Artikels 6, Absätze 3a, 10 und 11“, http//europa.eu/ 